\title{
ESTRATÉGIAS DE GESTÃO E (RE)ORGANIZAÇÃO DA REDE DE SAÚDE DE SOBRAL-CE NO ENFRENTAMENTO DA COVID-19
}

MANAGEMENT AND [RE]ORGANIZATION STRATEGIES OF SOBRAL-CE HEALTH NETWORK IN COPING WITH COVID-19

ESTRATEGIAS DE GESTIÓN Y (RE)ORGANIZACIÓN DE LA RED DE SALUd SOBRAL-CE PARA ENFRENTAR LA COVID-19

Regina Célia Carvalho da Silva ${ }^{1}$

Ivo Ferreira Gomes 2

Marcos Aguiar Ribeiro ${ }^{3}$

Larisse Araújo de Sousa ${ }^{4}$

Tarciana Ferreira Serafim 5

Viviane de Morais Cavalcante ${ }^{6}$

Palavras-chave: Infecções por Coronavírus; Serviços de Saúde; Capacidade de Resposta diante de Emergências.

Keywords: Coronavirus Infections; Health Services; Emergency Response Capacity.

Palabras clave: Infecciones por coronavirus; Servicios de salud; Capacidad de respuesta ante emergencias.

Submetido: $07 / 11 / 20$

Aprovado: $17 / 12 / 20$

Autor(a) para Correspondência: Regina Célia Carvalho da Silva R. Anaid Andrade, 373 Centro Sobral - CE CEP:62011-200 E-mail: reginacarvalho@sobral.ce. gov.br

\section{RESUMO}

Este estudo tem como objetivo compartilhar a experiência de gestão da crise e (re)organização da Rede de Atenção à Saúde de Sobral, Ceará, para o enfrentamento da Covid-19. Trata-se de um estudo descritivo, do tipo relato de experiência, elaborado no contexto da pandemia de Covid-19, no período de março a novembro de 2020. Foram desenvolvidas estratégias de gestão da crise por meio da composição de colegiados e a adoção de um modelo assistencial com enfoque familiar e comunitário. A experiência do sistema de saúde de Sobral evidencia a importância dos investimentos realizados ao longo dos anos e do fortalecimento da Atenção Primária à Saúde como a coordenadora do cuidado, bem como a ampliação das Redes de Atenção à Saúde com a intervenção e a incorporação de dois hospitais e a ampliação de leitos nos hospitais existentes. Assim, todo o processo de (re)organização foi ancorado em um modelo assistencial com enfoque familiar e comunitário, que considera as singularidades dos territórios, a participação social e a articulação intersetorial no enfrentamento da Covid-19.

1. Enfermeira. Doutoranda em Saúde Pública pela Universidade Federal do Ceará (UFC). Secretária de Saúde do município de Sobral - CE. Sobral, Ceará, Brasil. E-mail: reginacarvalho@sobral.ce.gov.br. ORCID: https://orcid. org/0000-0002-6124-8427

2. Graduado em Direito pela Universidade Federal do Ceará (UFC). Prefeito do município de Sobral - CE. E-mail: ivo@sobral.ce.gov.br. ORCID: https://orcid.org/0000-0001-8104-6091

3. Enfermeiro. Doutorando em Enfermagem pela Universidade Federal de São Paulo (UNIFESP). Coordenador de Vigilância do Sistema de Saúde e Interventor do Hospital Dr. Estevam (HDE), da Secretaria Municipal de Saúde de Sobral - CE. E-mail: marcosribeiro@sobral.ce.gov.br. ORCID: https://orcid.org/0000-0001-7299-8007

4. Enfermeira. Coordenadora da Atenção Primária à Saúde da Secretaria Municipal de Saúde de Sobral - CE. E-mail: larissesousa@sobral.ce.gov.br. ORCID: https://orcid.org/0000-0002-9575-8855

5. Odontóloga. Mestranda em Saúde da Família (UVA/Renasf). Coordenadora da Atenção Especializada e Interventora do Hospital de Campanha Dr. Francisco Alves, da Secretaria Municipal de Saúde de Sobral - CE (20172020). E-mail:tarcianaserafim@sobral.ce.gov.br. ORCID: https://orcid.org/0000-0002-3707-4842

6. Advogada e Contadora. Pós-graduada em Administração pela Faculdade Ateneu. Coordenadora Jurídica da Secretaria Municipal de Saúde de Sobral - CE. E-mail: vivianecavalcante@sobral.ce.gov.br. ORCID: https://orcid. org/0000-0002-7716-7920 


\section{ABSTRACT}

This study aims to share the experience of crisis management and [re]organization of the Health Care Network in Sobral, Ceará, to face Covid-19. This is a descriptive study, experience report type, developed in the context of the Covid-19 pandemics in the period from March to November 2020. Crisis management strategies were developed through the composition of collegiate bodies and the adoption of a care model focused on family and community. The experience of Sobral health system shows the importance of investments made over the years and the reinforcement of Primary Health Care as the coordinator of health care, as well as the expansion of Health Care Networks with the intervention and incorporation of two hospitals and the expansion of beds in existing hospitals. Thus, the entire process of [re]organization was anchored on a care model focused on family and community, which considers the uniqueness of territories, social participation and intersectoral articulation in facing Covid-19.

\section{RESUMEN}

Este estudio tiene como objetivo compartir la experiencia de gestión de la crisis y [re] organización de la Red de Atención a la Salud en Sobral, Ceará, en el combate a la Covid-19. Se trata de un estudio descriptivo, tipo de relato de experiencia, desarrollado en el contexto de la pandemia Covid-19 en el período de marzo a noviembre de 2020. Las estrategias de manejo de la crisis se desarrollaron a través de la composición de órganos colegiados y la adopción de un modelo de atención con un enfoque familiar y comunitario. La experiencia del sistema de salud de Sobral muestra la importancia de las inversiones realizadas a lo largo de los años y el fortalecimiento de la Atención Primaria de Salud como coordinadora de la atención, así como la expansión de las Redes de Atención en Salud con la intervención y la incorporación de dos hospitales y la ampliación de camas en hospitales existentes. Asi, todo el proceso de [re]organización estuvo anclado en un modelo de atención con enfoque familiar y comunitario, que considera las singularidades de los territorios, la participación social y la articulación intersectorial en el combate a la Covid-19.

\section{INTRODUÇÃ 0}

Em dezembro de 2019, foram registrados casos de pneumonia com causa desconhecida na cidade de Wuhan, província de Hubei, China. Assim, a partir do isolamento do material genético do vírus, foi constatado um novo betacoronavírus, inicialmente denominado 2019-nCoV pela Organização Mundial da Saúde (OMS). Recentemente, esse passou a ser chamado de SARS-CoV-2 (do inglês, Severe Acute Respiratory Syndrome Coronavirus 2) ${ }^{1-3}$.

Vale ressaltar que, apesar das infecções ocasionadas pelo coronavírus serem geralmente leves, nas epidemias por SARS-CoV, em 2002, e MERS-CoV (do inglês, Middle East Respiratory Syndrome), em 2012, mais de 10.000 pessoas foram infectadas, com taxas de mortalidade de $10 \%$ e $37 \%$, respectivamente 4 . 0 vírus SARS-CoV-2 é o causador da Covid-19 (do inglês, Coronavirus Disease 2019). A propagação da Covid-19 aconteceu rapidamente na China e, posteriormente, pacientes infectados foram identificados em outros países, principalmente na Europa, nos Estados Unidos, no Canadá e no Brasil ${ }^{5}$.

Em 3 de janeiro de 2020, o Ministério da Saúde (MS) do Brasil decretou, por meio da Portaria $n .^{\circ}$ 188/2020, o Estado de Emergência em Saúde Pública de Importância Nacional (ESPIN), em virtude da disseminação global da Covid-196. Em 11 de março de 2020, foi declarada pela OMS a condição de transmissão pandêmica da doença e, em março de 2020, foi declarado estado de transmissão comunitária em todo o território brasileiro, conforme Portaria n. ${ }^{454}$, do MS, de 20 de março de $2020^{7}$.

Nesse contexto, o Ministério da Saúde, a Secretaria Estadual de Saúde do Ceará e a Secretaria Municipal de Saúde de Sobral vêm adotando medidas de contenção do aumento do número de infecções por SARS-CoV-2.

No âmbito do estado do Ceará, o Governo Estadual publicou o Decreto $n .{ }^{\circ} 33.510$, de 16 de março de $2020^{8}$, adotando medidas significativas de contingenciamento da pandemia. Em Sobral, diante da responsabilidade municipal no enfrentamento ao novo coronavírus, a Prefeitura Municipal decretou, em 13 de março de 2020 (Decreto n. $\left.{ }^{\circ} 2.369 / 2020\right)^{9}$, estado de perigo público na rede hospitalar do município de Sobral; e, em 16 de março de 2020, estado de emergência municipal, conforme Decreto n. ${ }^{0} 2.371$ de $2020^{10}$.

Nessa perspectiva, o município de Sobral-CE reestruturou os serviços e as ações da Rede de Atenção à Saúde (RAS) com vistas ao desenvolvimento de 
medidas de vigilância, prevenção, controle e cuidado frente à Covid-19. Assim, este manuscrito tem como objetivo compartilhar a experiência de gestão da crise e (re)organização da RAS de Sobral-CE para o enfrentamento da Covid-19.

\section{METODOLOGIA}

Trata-se de um estudo descritivo, do tipo relato de experiência, desenvolvido no contexto do processo de (re)organização da Rede de Atenção à Saúde de Sobral-CE para o enfrentamento da Covid-19.

Sobral é um município do interior do estado do Ceará, localizado a 235 quilômetros da capital Fortaleza, com uma população de 208.935 habitantes, segundo estimativa do Instituto Brasileiro de Geografia e Estatística (IBGE), para o ano de 2019. Sobral integra uma rede assistencial hierarquizada e regionalizada do Sistema Único de Saúde (SUS), apresentando uma capacidade instalada para o desenvolvimento e a realização de serviços de diversas densidades tecnológicas, o que o torna referência para a Macrorregião Norte do estado do Ceará, composta por 55 municípios, abrangendo uma população de aproximadamente 1.606 .608 habitantes ${ }^{11}$.

0 modelo assistencial e de cuidado à saúde desse município tem a Saúde da Família (SF) como estratégia prioritária para a reorganização da RAS, de maneira que apresenta uma proporção de cobertura assistencial estimada de $100 \%$, de acordo com dados da Coordenação Geral de Informação da Atenção Primária do Departamento de Saúde da Família da Secretaria de Atenção Primária - CGIAP/DESF/SAPS, do Ministério da Saúde ${ }^{12}$. Para isso, são 70 equipes de Saúde da Família (eSF) distribuídas em 37 Centros de Saúde da Família (CSF), bem como seis equipes de Núcleo Ampliado de Saúde da Família e Atenção Básica (Nasf-AB), 50 equipes de saúde bucal, três equipes multiprofissionais de Atenção Domiciliar e duas academias da saúde ${ }^{11}$.

\section{"O COESP-S foi legitimado por meio de portaria municipal e configurou- se com um potente espaço de planejamento e construção coletiva"}

No que concerne à sistematização de informações para a construção do relato dessa experiência, utilizaram-se relatórios e anotações pessoais com relatos das observações, sentimentos e vivências realizados no período de março a novembro de 2020 .

\section{RESULTADOS E DISCUSSÃO}

\section{Estratégias Colegiadas de Gestão da Crise}

Em janeiro de 2020, o Sistema de Saúde de Sobral se deparou com o primeiro caso suspeito de contaminação por Covid-19. Consistia em um adulto jovem com histórico de viagem para a China e que apresentou sintomatologia respiratória e procurou um hospital privado do município de Sobral. Posteriormente, foi encaminhado para um hospital público, onde permaneceu em isolamento até o resultado do exame que descartou o caso.

Esse primeiro caso evidenciou a necessidade do Sistema de Saúde de Sobral de aproximar diferentes atores no processo de planejamento para a (re)organização da RAS no enfrentamento da Covid-19. Assim, buscou-se avaliar o nível de resposta do sistema de saúde nesse primeiro caso e a partir de então aprender com as fragilidades e as potencialidades identificadas.

Essa capacidade crítica possibilitou a percepção e a problematização da situação vivenciada. Nesse sentido, o planejamento e a avaliação propiciaram a instauração de novas aprendizagens e as revisões das intervenções abordadas, bem como a qualificação da comunicação entre os trabalhadores e os gestores do SUS. As práticas de planejamento, gestão e avaliação, não somente na perspectiva metodológica ou técnica, construíram-se como meios e processos para o compartilhamento de poder, desalienação dos sujeitos, compromisso efetivo e eficácia ${ }^{13}$.

Desse modo, foi constituído no município um colegiado no qual as suas discussões possibilitaram a construção da primeira edição do Plano de Contingência para Infecção Humana pelo novo coronavírus (Covid-19). Dentre as ações previstas no Plano, foi constituído o Centro de Operações de Emergências em Saúde Pública de Sobral (COESP-S).

A construção do plano de contingência viabilizou - planejamento das atividades relacionadas ao enfrentamento da pandemia, de forma a garantir a aquisição, produção, armazenamento, distribuição de equipamentos e insumos, bem como de serviços e ações coerentes com cada fase da pandemia. 
A efetivação do planejamento reconhece a pandemia como um fenômeno complexo que exige a ressignificação dos serviços e ações ofertados e as várias dimensões e suas interfaces com as evidências científicas, saberes técnicos, práticas e movimentos sociais ${ }^{14}$.

0 COESP-S foi legitimado por meio de portaria municipal e configurou-se com um potente espaço de planejamento e construção coletiva por meio de ações de resposta à situação de emergência em Saúde Pública, bem como de mobilização e articulação intersetorial.

Esse colegiado apresentava a seguinte composição: Gabinete do Prefeito; Secretaria da Saúde; Coordenação Administrativo-financeira; Coordenadoria de Políticas e Planejamento de Atenção à Saúde (COPPAS); Assessoria de Comunicação; Coordenadoria de Vigilância em Saúde; Célula de Vigilância Epidemiológica em Saúde; Célula de Vigilância Sanitária; Célula do Centro de Referência em Saúde do Trabalhador (CEREST); Coordenadoria de Vigilância do Sistema de Saúde; Célula de Auditoria e Regulação em Saúde; Coordenadoria da Atenção Primária à Saúde; Assistente Especial da Atenção Primária à Saúde; Coordenadoria da Atenção Especializada; Centro de Referência em Infectologia de Sobral (CRIS); Central de Abastecimento Farmacêutico (CAF); Escola de Saúde Pública Visconde de Saboia (ESPVS); Hospital Santa Casa de Misericórdia de Sobral; Hospital Regional Norte; Hospital Unimed; Unidade de Pronto Atendimento; Secretaria de Educação; Secretaria de Cultura, Juventude, Esporte e Lazer (SECJEL); Secretaria de Urbanismo e Meio Ambiente (SEUMA); Universidade Estadual Vale do Acaraú (UVA); Universidade Federal do Ceará (UFC); Grupo Luciano Feijão; Centro Universitário INTA (UNINTA); Instituto Federal de Educação, Ciência e Tecnologia do Ceará (IFCE); Grendene S/A; Grupo Votorantim; Câmara de Vereadores de Sobral; Ministério Público (MP); Organização de Advogados do Brasil $(O A B)$; Defensoria Pública (DP); Cadeia Pública; Conselho Municipal de Saúde de Sobral (CMSS); Superintendência da Região Norte de Sobral (SRNOR).

Coube ao COESP-S o papel de acompanhar as orientações da Organização Mundial da Saúde, Ministério da Saúde, Agência Nacional de Vigilância Sanitária (Anvisa), Secretaria Estadual da Saúde e outras instituições pertinentes, bem como os estágios da pandemia e os níveis de ativação de resposta. Além disso, esse coletivo buscou avaliar

\section{"A intersetorialidade é premente para 0 desenvolvimento das políticas públicas"}

as medidas prioritárias de enfrentamento por meio do acompanhamento da situação de saúde e das intervenções realizadas, além de mobilizar os diversos atores da sociedade por meio de significativas articulações intersetoriais que promoveram o engajamento da sociedade através de doações, mudanças no processo produtivo de indústrias locais para a confecção de Equipamentos de Proteção Individual (EPIs), apoio de pesquisadores das Instituições de Ensino Superior, fortalecimento de estratégias de comunicação para estímulo ao isolamento social, entre outras atividades.

A intersetorialidade é premente para 0 desenvolvimento das políticas públicas e fundamental para intensificar resultados nos âmbitos social e da saúde. Nessa perspectiva, a concepção de intersetorialidade precisa atravessar o cotidiano das políticas públicas por meio da construção de diferentes modos e conexões, de maneira a promover uma ligação orgânica com vistas ao alcance de maior integralidade e equidade ${ }^{15}$.

0 exercício da intersetorialidade exige não apenas o desempenho de gestores e trabalhadores, sendo necessária a construção de espaços para o diálogo e práxis intersetorial a partir do desenvolvimento de políticas públicas que orientem e promovam sua efetivação ${ }^{16}$. A institucionalização do COESP-S representa o movimento de encontro de atores estratégicos para o planejamento, avaliação e mobilização de setores governamentais, não governamentais e sociedade civil.

Esse movimento intersetorial foi capaz de mobilizar e configurar o modo de produção das indústrias locais de Sobral para a confecção de EPI, de forma coordenada, pela equipe da Secretaria da Saúde e representantes do COESP-S. 0 processo de "reconversão industrial" ou "reconversão produtiva" coordenado pelo Estado para a utilização emergencial de indústrias para a produção de materiais necessários na pandemia foi refletido pelo Departamento Intersindical de Estatística e Estudos Econômicos 
(DIEESE), que considera que o Estado deve ser o principal indutor de formulação de políticas públicas orientadas à reconversão industrial, assumindo compromisso no financiamento e na garantia de aquisição de materiais e equipamentos do Complexo Industrial da Saúde ${ }^{17}$.

Apesar dessa exitosa experiência em Sobral, verifica-se, em âmbito nacional, uma ausência do Governo Federal nesse processo de indução da reconversão industrial ${ }^{17}$, como também o abandono e o estímulo a o acirramento de conflitos entre os entes federados e os segmentos da sociedade, atravessado por um discurso negacionista que prejudicou a condução nacional no enfrentamento da pandemia ${ }^{14}$.

Além do COESP-S, foi constituído, no âmbito da Secretaria da Saúde, o Comitê de Crise, destinado à deliberação de medidas estratégicas de enfrentamento da Covid-19, bem como às articulações com o COESP-S e o Controle Social. Esse coletivo foi formado por gestores e técnicos da Secretaria da Saúde que, diariamente, reuniam-se em um escritório de crise para o planejamento, a deliberação e a avaliação das ações em desenvolvimento.

\section{(Re) organização da RAS}

No contexto da pandemia de Covid-19, os profissionais de saúde que integram a Atenção Primária à Saúde (APS) precisaram adequar seus serviços e ações de modo a incorporar o atendimento remoto, bem como fortalecer as ações de prevenção do contágio e os cuidados a usuários com suspeita ou confirmação de Covid-19, com enfoque em uma abordagem familiar, comunitária e territorial.

A partir da compreensão que a APS é a ordenadora do cuidado e a porta de entrada preferencial para o desenvolvimento de ações e serviços ofertados pela RAS, em Sobral, ela ocupou o papel central no enfrentamento da Covid-19 a partir da necessidade imediata de capilarização das informações e ações para as comunidades ${ }^{18}$. Nesse sentido, a linha de cuidado foi construída tendo como referência a APS como gestora do cuidado na RAS.

Os Centros de Saúde da Família (CSF) adaptaram a carteira de serviços e ações ofertados com o objetivo de evitar aglomerações e garantir a proteção dos usuários e famílias dos seus respectivos territórios. As estruturas dos CSF foram adaptadas com a reorganização da porta de entrada e a triagem em tendas na área externa da unidade de saúde, propositalmente implantadas para esse fim.

\section{"Os trabalhadores da APS precisaram transformar suas práticas, aprender com os desafios diários $e$ incorporar novas tecnologias de cuidado"}

Outrossim, os trabalhadores tiveram suporte por meio de ações de educação permanente e ampliação das estratégias de acompanhamento dos usuários por meio de recursos tecnológicos.

A Educação Permanente é um campo de ação estratégica que passou de um conceito pedagógico a signo da educação no SUS, ou seja, carrega ressignificação da práxis por meio da recomposição das práticas de atenção à saúde, de modo que a integralidade e a gestão participativa constituem-se como eixos fundamentais do seu desenvolvimento ${ }^{19}$. Nesse contexto, os trabalhadores da APS precisaram transformar suas práticas, aprender com os desafios diários e incorporar novas tecnologias de cuidado no enfrentamento da pandemia.

Assim, a APS realizou o processo de identificação de sintomáticos respiratórios e, a partir de então, orientava e monitorava o isolamento domiciliar, bem como a evolução dos sintomas e a articulação com postos de coleta de exames de biologia molecular (RT-PCR em tempo real, detecção do vírus SARSCoV-2) e imunocromatográficos (teste rápido).

Foram acompanhados os usuários do seu território sanitário e, ao identificar a necessidade de compartilhamento do cuidado em rede com os demais serviços, dialogava-se com a atenção especializada para a realização de exames de imagem e com os hospitais e Unidades de Pronto Atendimento (UPA), para o processo de avaliação/internação. Além disso, os CSF articularam-se com o Plantão Epidemiológico $24 \mathrm{~h}$, que se constituiu como um canal de diálogo para os usuários do SUS. 0 plantão epidemiológico articulou-se com a Central de Monitoramento da Covid-19 e o Comitê de Crise, que compartilhavam situações específicas de usuários com os gerentes da APS, os quais planejavam e desenvolviam junto às equipes estratégias singulares de monitoramento e cuidado aos usuários inseridos nos territórios.

Para esse monitoramento, os Agentes Comunitários de Saúde (ACS) permaneceram atuando nos territórios com visitas peridomiciliares; nestas, forneceram 
orientações acerca do isolamento domiciliar, medidas de prevenção da doença e identificação de sinais de complicação. Para a realização das visitas, foram destinados Equipamentos de Proteção Individual (EPIs) e processos formativos relacionados à Covid-19. Os Agentes de Combate às Endemias (ACE) permaneceram nos territórios atuando na prevenção das arboviroses.

A reorganização do processo de trabalho do ACS, na pandemia, precisou considerar a orientação comunitária e a competência cultural como eixos basilares, de modo a não permitir que esses eixos fossem substituídos por outras racionalidades, tais como trabalhos somente burocráticos e não efetivos ao cuidado em saúde. A práxis do ACS deve considerar, ainda, a perspectiva da educação em saúde de maneira construtivista, com o desenvolvimento de estratégias de comunicação culturalmente acessíveis e não meramente prescritivas, de modo a não atribuir aos usuários e famílias a culpa pela exposição. Ressalta-se também a necessidade de garantia de Equipamentos de Proteção Individual e outras condições de trabalho, coerentes com cenário de pandemia de Covid $-19^{20}$.

Além disso, buscou-se reorganizar o processo de dispensação de medicamentos de pacientes com condições crônicas, possibilitando um maior aprazamento no processo de renovação de receitas. 0 s atendimentos presenciais foram priorizados para os usuários com condições crônicas descompensadas e gestantes. Ressalta-se que esses grupos eram atendidos com criteriosas medidas de prevenção da contaminação pela Covid-19 e acessavam o CSF por entradas específicas, diferentes daquelas utilizadas por pacientes sintomáticos respiratórios.

Os investimentos nas estruturas dos CSF de Sobral, nos últimos anos, foram fundamentais para a reorganização da APS no enfrentamento da Covid-19, uma vez que a maioria dos CSF apresentam amplo espaço, com ambiência que permite uma boa ventilação e a organização de entradas diferentes para os usuários com sintomas respiratórios e os demais usuários. Ademais, os investimentos realizados nos últimos anos na qualificação de processos de trabalho também foram relevantes para o enfrentamento da pandemia no contexto da APS. Ressaltam-se o monitoramento e a territorialização, com 0 desenvolvimento de instrumentos de estratificação de risco de pacientes com Hipertensão Arterial Sistêmica (HAS), Diabetes mellitus e de gestantes, estas, com suporte e acompanhamento

\section{"Os investimentos nas estruturas dos CSF de Sobral, nos últimos anos, foram fundamentais para a reorganização da APS no enfrentamento $d a$ Covid-19"}

multiprofissionais pela Estratégia Trevo de Quatro Folhas.

As ações e serviços de saúde bucal foram suspensos com a finalidade de evitar a disseminação do vírus no momento de execução dos procedimentos odontológicos, mantendo-se 0 atendimento às urgências odontológicas que estão sendo referenciadas para a UPA. 0s profissionais da saúde bucal ressignificaram sua práxis de maneira a contribuir com os serviços do Fast-Track Covid-19 na fase de identificação (nível técnico) e de avaliação dos sintomas e notificação (nível superior), bem como no processo de monitoramento e organização dos serviços no âmbito da APS.

Em relação à estrutura da rede de atenção especializada de Sobral, o Centro de Especialidades Odontológicas Municipal e o Centro de Especialidades Odontológicas Regional tiveram suas atividades suspensas nas fases de contenção e mitigação (epidemia localizada e aceleração). Nessas fases, os serviços de saúde bucal restringiram-se aos serviços de urgência odontológica realizados na UPA. Os profissionais do Centro de Especialidades Odontológicas (CEO) Municipal foram incorporados à APS com vistas a fortalecer as atividades de monitoramento de pacientes suspeitos e confirmados de Covid-19.

A maior parte das atividades de atendimento presencial do Centro de Especialidades Médicas (CEM) foi suspensa, todavia foram mantidos os pré-natais de Alto Risco e as ultrassonografias obstétricas, com o objetivo de constituir-se como uma retaguarda para a APS. As demais especialidades iniciaram o processo de matriciamento remoto com a APS, de maneira que os médicos deste nível de atenção compartilharam os casos com os médicos especialistas, que matriciaram estes casos apoiando na definição dos projetos terapêuticos.

A Policlínica também teve a maior parte das suas atividades suspensas, porém foram mantidos 
os pré-natais de Alto Risco, o ambulatório do pé diabético e os serviços de imagem (raio-X e tomografia computadorizada). Além disso, a Policlínica estruturou um plano de ação para suporte à UPA e aos hospitais na realização de tomografia computadorizada e raio- $X$ dos pacientes com Covid-19.

0 Centro de Reabilitação de Sobral tipo II (CER-II) teve as suas atividades suspensas nas fases de contenção e mitigação. Os profissionais fisioterapeutas do CER-II foram deslocados para - Hospital Dr. Estevam (HDE), no enfrentamento da Covid-19, e os demais profissionais foram direcionados para o fortalecimento das atividades de monitoramento de casos suspeitos/confirmados no contexto da APS.

0 Centro de Referência em Infectologia (CRIS) continuou com o desenvolvimento de suas atividades, todavia com a incorporação de orientações remotas por meio de contato telefônico e a redução dos atendimentos presenciais, uma vez que foram priorizados os casos mais complexos que necessitavam de avaliação do infectologista e da equipe multiprofissional.

Os antirretrovirais Tenofovir $300 \mathrm{mg}+$ Lamivudina $300 \mathrm{mg}$; Dolutegravir $50 \mathrm{mg}$; Tenofovir $300 \mathrm{mg}+$ Lamivudina $300 \mathrm{mg}$ + Efavirenz $300 \mathrm{mg}$; Ritonavir $100 \mathrm{mg}$; Atazanavir $300 \mathrm{mg}$; Zidovudina $300 \mathrm{mg}+$ Lamivudina $300 \mathrm{mg}$; Raltegravir $400 \mathrm{mg}$; Tenofovir $300 \mathrm{mg}+$ Emtricitabina $200 \mathrm{mg}$ foram dispensados para 60 dias com o objetivo de reduzir a quantidade de pacientes na farmácia satélite do CRIS; todavia, os pacientes foram acompanhados remotamente acerca de aspectos relacionados à adesão e efeitos adversos. A dispensação das outras medicações foi aprazada normalmente para 30 dias.

A Rede de Atenção Integral em Saúde Mental (RAISM) de Sobral é composta pelo Centro de Atenção Psicossocial Damião Ximenes Lopes (CAPS-Geral), Centro de Atenção Psicossocial Álcool e Outras Drogas Francisco Hélio Soares (CAPS AD), Residência Terapêutica (RT), leitos psiquiátricos no Hospital Dr. Estevam e pela Unidade de Acolhimento (UA). Os CAPS tiveram suas atividades presenciais restritas e o funcionamento em horário reduzido nas fases de contenção e mitigação com o objetivo de diminuir a disseminação da Covid-19.

Os profissionais da RAISM também realizaram, junto aos docentes e residentes da Residência Multiprofissional em Saúde da Família (RMSF) e Residência Multiprofissional em Saúde Mental

\section{"Profissionais foram direcionados para o fortalecimento das atividades de monitoramento de casos suspeitos/confirmados no contexto da APS"}

(RMSM), vinculadas à Escola de Saúde Pública Visconde de Saboia, atendimentos remotos e plantões de escuta para profissionais da saúde e usuários, com o suporte dos psicólogos e assistentes sociais residentes.

A Unidade de Acolhimento ressignificou seu perfil de atendimento, de modo que passou a ser uma referencia para o isolamento social de pessoas em situação de rua com sintomas respiratórios e demais usuários com dificuldade de isolamento no seu domicílio. Assim, a referida unidade manteve o funcionamento durante $24 \mathrm{~h}$, sob a gestão da Coordenadoria de Políticas sobre Drogas, com uma equipe multiprofissional e o suporte da RMSM. Além disso, a UA articulou-se com equipes do Centro de Referência Especializado para População em Situação de Rua (Centro Pop), Centro de Referência da Assistência Social (CRAS) e Centro de Referência Especializado de Assistência Social (CREAS) no processo de proteção de pessoas em situação de rua.

0 Serviço de Atendimento Móvel de Urgência (SAMU 192) foi fundamental para o suporte às urgências e emergências e para o translado de pacientes suspeitos/confirmados de Covid-19 para os hospitais de referência e demais serviços de diagnósticos.

A UPA constituiu-se como uma das portas de entrada para as urgências e emergências, inclusive as relacionadas à Covid-19. Os pacientes eram avaliados e, se necessário, ficavam em observação na UPA enquanto aguardavam a regulação para os hospitais de referência.

No que se refere à rede hospitalar, diante dessa responsabilidade de Sobral, em 13 de março de 2020 foi declarado estado de perigo público na rede hospitalar do município e decretada a intervenção municipal por modalidade de requisição do prédio e de todas as instalações físicas do Hospital Dr. Estevam Ponte, por meio do Decreto n. ${ }^{0} 2.369^{9}$. A partir de então, o HDE integrou a rede de hospitais 
no enfrentamento da doença pelo SARS-CoV-2 para casos de baixa e média complexidade. Além disso, o HDE constituiu-se como Centro de Referência para Testagem RT-PCR em tempo real, detecção do vírus SARS-CoV-2.

Em 20 de março de 2020, foi decretada a intervenção municipal por modalidade de requisição do prédio e de todas as instalações físicas do Hospital Dr. Francisco Alves (HDA). Nessa estrutura física, foi instalado o hospital de campanha da Covid-19, com o acesso aos leitos clínicos e leitos de Unidade de Terapia Intensiva (UTI) de referência para a Macrorregião Norte do estado do Ceará.

Além disso, foram ampliados os leitos clínicos e de UTI no Hospital Santa Casa de Misericórdia de Sobral, hospital filantrópico prestador do SUS, contratualizado com a Secretaria da Saúde de Sobral. Ampliaram-se, ainda, os leitos clínicos e de UTI no Hospital Regional Norte (HRN), sob a gestão da Secretaria da Saúde do Estado do Ceará. Na fase de aceleração da doença, o HRN ampliou mais leitos clínicos com a abertura de um hospital de campanha vinculado à sua estrutura física.

0 Hospital do Coração de Sobral destinou leitos para pacientes com condições cardíacas que apresentavam sintomatologia respiratória. Ademais, a Secretaria da Saúde estimulou a criação e monitorou os leitos de UTI privados implantados no Hospital UNIMED.

A ampliação da rede hospitalar para o enfrentamento da Covid-19 é importante, principalmente em locais onde historicamente existem significativos vazios assistenciais, como também naqueles onde já se observa uma pressão nos hospitais existentes devido à grande demanda ${ }^{21}$. Reflete-se também que, para o processo de ampliação da rede hospitalar, fazse necessário planejar de forma regionalizada e garantir a definição de uma linha de cuidado que possibilite o acesso e a integralidade, de modo a levar em consideração a APS como coordenadora e gestora do cuidado em rede.

\section{CONCLUSÃO}

A experiência de (re)organização da RAS de Sobral evidencia a importância dos investimentos na estrutura e no desenvolvimento de processos de trabalho realizados ao longo dos anos e do fortalecimento da APS como coordenadora do cuidado, bem como a ampliação da RAS com a intervenção e incorporação de dois hospitais e a ampliação de

\section{"Viver a gestão do \\ SUS nesse período de pandemia foi desafiador, pois o vivido foi atravessado por medos, anseios e incertezas"}

leitos nos hospitais existentes.

Nesse sentido, todo o processo de (re) organização foi ancorado em estratégias colegiadas e colaborativas de gestão da crise por meio da composição do COESP-S e do Comitê de Crise, como também na adoção de um modelo assistencial com enfoque familiar e comunitário, que considera as singularidades dos territórios, a participação social e a articulação intersetorial no enfrentamento da Covid-19.

Assim, as memórias e os sentimentos vividos nesse período de intenso trabalho na gestão da crise trazem importantes reflexões acerca da necessidade de fortalecimento da democracia e defesa do SUS. Além disso, colocam em destaque o papel de gestor do SUS na construção de políticas públicas capazes de garantir respostas rápidas, resolutivas e coerentes com o atual contexto de enfrentamento da pandemia.

Viver a gestão do SUS nesse período de pandemia foi desafiador, pois o vivido foi atravessado por medos, anseios e incertezas. Todavia, a coragem de enfrentar os desafios e a importância de mobilizar mentes e corações no processo de defesa da vida impulsionaram o coletivo ao trabalho colaborativo que se ressignificava em cada fase da pandemia e que potencializava a formação de fluxos e linhas de conexão entre trabalhadores, serviços e ações em saúde.

\section{CONTRIBUIÇÃO DOS AUTORES}

Regina Célia Carvalho da Silva contribuiu com redação, análise e revisão crítica do manuscrito. Ivo Ferreira Gomes contribuiu com redação, análise e revisão crítica do manuscrito. Marcos Aguiar Ribeiro contribuiu com redação, análise e revisão crítica do manuscrito. Larisse Araújo de Sousa contribuiu com redação, análise e revisão crítica do manuscrito. Tarciana Ferreira Serafim contribuiu com redação, análise e revisão crítica do manuscrito. Viviane de 
Morais Cavalcante contribuiu com redação, análise e revisão crítica do manuscrito.

\section{REFERÊNCIAS}

1. Chen Y, Guo Y, Pan Y, Zhao ZJ. Structure analysis of the receptor binding of 2019-nCoV. Biochem Biophys Res Commun [serial on the internet]. 2020 [cited 2020 Nov 28];525(1):135-40. Available from: https://www.sciencedirect.com/science/article/pii/ $\underline{\text { S0006291X20303399 }}$

2. Zhang J-J, Dong $X$, Cao Y-Y, Yuan Y-D, Yang $Y-B, Y a n Y-Q$, et al. Clinical characteristics of 140 patients infected with SARS-CoV-2 in Wuhan, China. Allergy [serial on the internet]. 2020 [cited 2020 Nov 11];75(5):1730-41. Available from: https://onlinelibrary.wiley.com/doi/10.1111 /all.14238

3. Beeching NJ, Fletcher TE, Fowler R. COVID-19. BMJ Best Practice [serial on the internet]. 2020 [cited 2020 Nov 11];1(1):1-55. Available from: https://www.spg.pt/wp-content/uploads/ 2020/04/BMJ-Covid-Best-Practice.pdf

4. Huang C, Wang $Y$, Li X, Ren L, Zhao J, Hu Y, et al. Clinical features of patients infected with 2019 novel coronavirus in Wuhan, China. Lancet [serial on the internet]. 2020 [cited 2020 Nov 09];395(10223):497-506. Available from: https:// www.thelancet.com/journals/lancet/article/ PIIS0140-6736(20)30183-5/fulltext

5. Brasil. Diretrizes para diagnóstico e tratamento da COVID-19. Brasília (DF): Editora do Ministério da Saúde; 2020.

6. Brasil. Portaria $n^{\circ} 188$, de 3 de fevereiro de 2020. Declara Emergência em Saúde Pública de importância Nacional (ESPIN) em decorrência da Infecção Humana pelo novo Coronavírus (2019-nCoV) [document on the internet]. Diário Oficial da União, Brasília (2020 Feb 3 [cited 2020 Nov 15]). Available from: https:// www.in.gov.br/web/dou/-/portaria-n-188-de-3-defevereiro-de-2020-241408388

7. Brasil. Portaria $n^{\circ} 454$, de 20 de março de 2020. Declara, em todo o território nacional, o estado de transmissão comunitária do coronavírus (COVID-19) [document on the internet]. Diário Oficial da União, Brasília (2020 Mar 20 [cited 2020 Nov 16]). Available from: https://www.in.gov .br/en/web/dou/-/portaria-n-454-de-20-de-marcode-2020-249091587

8. Ceará. Decreto n 33.510, de 16 de março de 2020. Decreta situação de emergência em saúde e dispõe sobre medidas para enfrentamento e contenção da infecção humana pelo novo coronavírus [document on the internet]. Diário Oficial do Estado do Ceará, Fortaleza (2020 Mar 16 [cited 2020 Nov 16]). Available from: https://coronavirus.ceara. gov.br/project/decreto-no-33-510-de-16-de-marcode-2020/

9. Sobral. Decreto $n^{0} 2.369 / 2020$. Decreta estado de perigo público na rede hospitalar do município de Sobral e estabelece medidas para enfrentamento do novo coronavírus (COVID- 19), e dá outras providências [document on the internet]. Diário Oficial de Sobral, Sobral (2020 Mar 20 [cited 2020 Nov 16]). Available from: http://www.sobral. ce.gov.br/diario/public/files/diario/4f532ab2361 eeece2b08d4dde8012de1.pdf

10. Sobral. Secretaria da Saúde. Decreto $n^{\circ} 2.371$ de 2020. Decreta estado de emergência no âmbito do município de Sobral e estabelece medidas para enfrentamento do novo coronavírus (COVID19), e dá outras providências [document on the internet]. Diário Oficial de Sobral, Sobral (2020 Mar 20 [cited 2020 Nov 16]). Available from: http://www.sobral.ce.gov.br/diario/public/files/di ario/9bf7ba4ae2931bdf14b3a48b5240d141.pdf

11. Sobral. Plano de contingência diante da infecção humana pelo Novo Coronavírus (COVID-19) da Secretaria da Saúde de Sobral. 3. ed. Sobral (CE): Secretaria da Saúde; 2020.

12. Brasil. e-Gestor Atenção Básica. Cobertura da Atenção Básica [home-page on the internet]. 2020 [cited 2020 Nov 28]. Available from: https://egestorab.saude.gov.br/paginas/ acessoPublico/relatorios/relHistoricoCoberturaAB. $\underline{x h t m l}$

13. Furtado JP, Campos GWS, Oda WY, OnockoCampos R. Planejamento e Avaliação em Saúde: entre antagonismo e colaboração. Cad. Saúde Pública [serial on the internet]. 2018 [cited 2020 Nov 18];34(7):e00087917. Available from: http:// www.scielo.br/scielo.php?script $=$ sci arttext\&pid $=$ S0102311X2018000705004\&lng =en

14. Souto LRF, Travassos C. Plano Nacional de Enfrentamento à Pandemia da Covid-19: construindo uma autoridade sanitária democrática. Saúde Debate [serial on the internet]. 2020 [cited 2020 Nov 28];44(126):587-89. Available from: http:// www.scielo.br/scielo.php?script $=s c i \quad$ arttex t\&pid $=$ S0103-11042020000300587\&lng $=e n$

15. Carmo ME, Guizardi FL. Desafios da intersetorialidade nas políticas públicas de saúde e assistência social: uma revisão do estado da arte. Physis [serial on the internet]. 2017 [cited 2020 Nov 21];27(4):1265-86. Available from: http://www.scielo.br/scielo.php?script=sci arttex t\&pid $=$ S0103-73312017000401265\&lng $=$ en

16. Dias MSA, Parente JRF, Vasconcelos MI0, Dias FAC. Intersetorialidade e Estratégia Saúde da 
Família: tudo ou quase nada a ver?. Cien Saúde Colet [serial on the internet]. 2014 [cited 2020 Nov 10];19(11):4371-82. Available from: http://www. scielo.br/scielo.php?script $=$ sci arttext \&pid=S1 413-81232014001104371\&lng =en

17. Departamento Intersindical de Estatística e Estudos Econômicos. Nota Técnica No 238 Reconversão industrial em tempos de Covid-19: o papel dos governos para salvar vidas [document on the internet]. São Paulo: DIEESE; 2020 [cited 2020 Nov 10]. Available from: http://www.epsjv.fiocruz. $\mathrm{br} / \mathrm{sites} / \mathrm{default} /$ files/files/DIEESE\%20N0TA\%20 T\%C $3 \% 89$ CN ICA \% $20238 \% 20$ reconversao $\% 20$ industrial.pdf.pdf

18. Ribeiro MA, Júnior DGA, Cavalcante ASP, Martins $A$, de Sousa L, Carvalho R, Cunha ICKO. (RE) Organização da Atenção Primária à Saúde para o enfrentamento da COVID-19: Experiência de SobralCE. APS em ver [serial on the internet]. 2020 [cited 2020 Nov 10];2(2):177-88. Available from: https:// apsemrevista.org/aps/article/view/125

19. Ceccim RB. Emergência de um “campo de ação estratégica": ordenamento da formação e educação permanente em saúde. Sanare [serial on the internet]. 2019 [cited 2020 Nov 18];18(1):68-80. Available from: https://sanare.emnuvens.com.br/ sanare/article/view/1307

20. Maciel FBM, Santos HLPC, Carneiro RAS, Souza EA, Prado NMBL, Teixeira CFS. Agente comunitário de saúde: reflexões sobre o processo de trabalho em saúde em tempos de pandemia de Covid-19. Cien Saúde Colet [serial on the internet]. 2020 [cited 2020 Nov 12];25(Suppl 2):4185-95. Available from: http://www.scielo.br/scielo.php?script=sci arttext\&pid $=$ S1413-81232020006804185\&lng $=e n$

21. Noronha KVMS, Guedes GR, Turra CM, Andrade MV, Botega L, Nogueira D et al. Pandemia por COVID-19 no Brasil: análise da demanda e da oferta de leitos hospitalares e equipamentos de ventilação assistida segundo diferentes cenários. Cad. Saúde Pública [serial on the internet]. 2020 [cited 2020 Nov 13];36(6):e00115320. Available from: $\quad$ http://www.scielo.br/scielo.php?script $=\mathrm{s} c \mathrm{i} \quad \mathrm{art} \mathrm{t} \times \mathrm{t} \& \mathrm{p} \mathrm{i} d=\mathrm{s} 010102-$ $311 \times 2020000605004 \& \operatorname{lng}=$ en
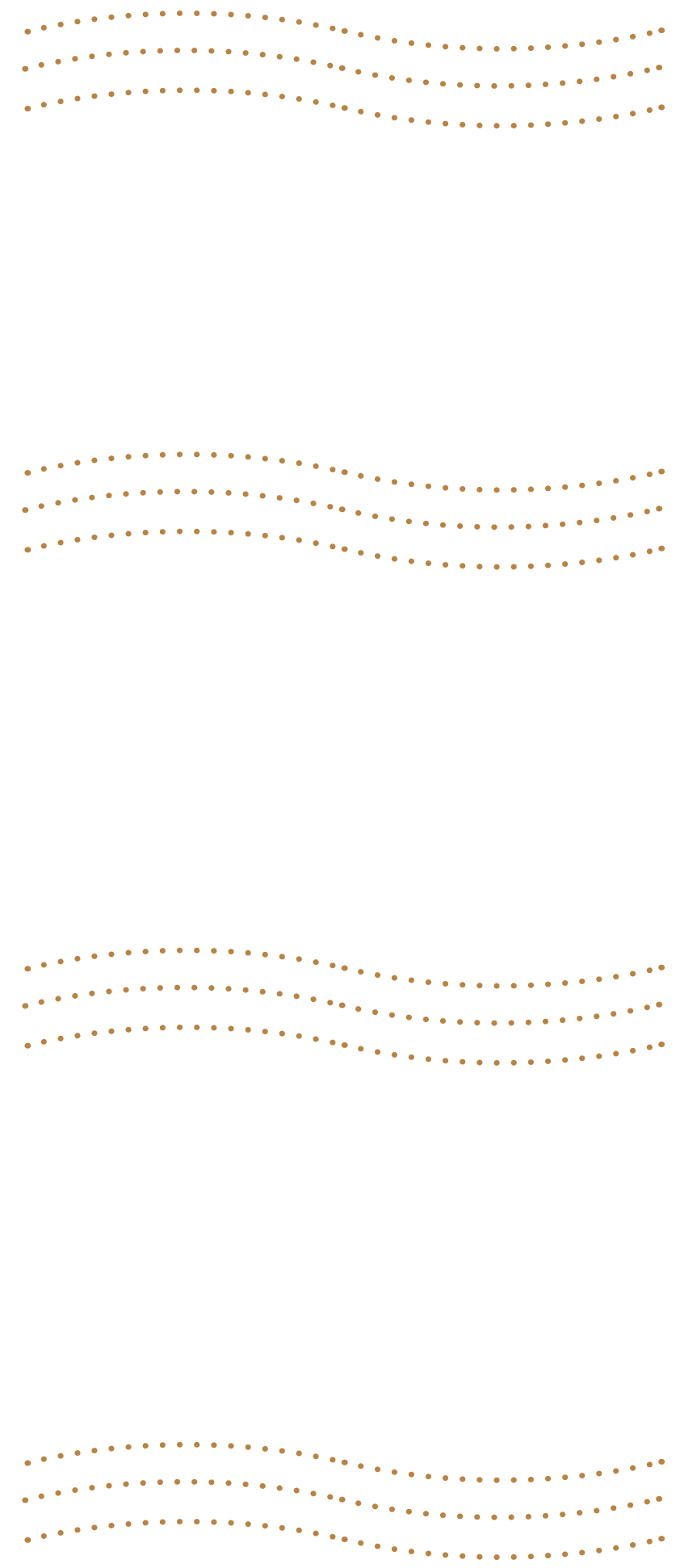